\title{
УДК 535.361 \\ Применение метода матриц формы для моделирования рассеивающих свойств пылевых частиц кометных атмосфер
}

\author{
Д.В. Петров ${ }^{1}$, Н.Н. Киселев ${ }^{1}$, Е.А. Жужулина ${ }^{2}$ \\ 1 ФГБУН “Крымская астрофизическая обсерватория РАН”, Научный, Крым, 298409 \\ dvp@craocrimea.ru \\ 2 Крымский федеральный университет им. В.И. Вернадского, пр-кт Академика Вернадского, 4, \\ Симферополь, Крым, 295007 \\ iguana-sev@mail.ru
}

Поступила в редакцию 15 ноября 2017 г.

\begin{abstract}
Аннотация. Современные задачи рассеяния электромагнитных волн частицами, сравнимыми с длиной волны, часто решаются с помощью метода расширенных граничных условий. В принципе, этот метод может применяться для изучения рассеяния объектами произвольной формы. Однако зачастую расчеты довольно сложны и требуют больших затрат компьютерного времени. В связи с этим упрощение и ускорение подобных расчетов представляется очень важной задачей. Разработанный нами метод матриц формы (Sh-matrix method) позволяет производить эффективное вычисление рассеивающих свойств частиц произвольной формы как с произвольным размерным параметром, так и с произвольным показателем преломления $m_{0}$. Данный метод применим для изучения кометных атмосфер, а также любых реголитоподобных сред. Проведено моделирование рассеивающих свойств кометной пыли с использованием случайных гауссовских частиц, состоящих из органики. На основании расчетов фазовой зависимости степени линейной поляризации подобраны параметры, наилучшим образом воспроизводящие наблюдательные данные кометы Галлея. Установлено, что органические частицы достаточно хорошо описывают значение угла инверсии и поляриметрического наклона, однако величину и положение минимума отрицательной ветви такие частицы воспроизвести не могут. На основании этого сделан вывод о необходимости рассматривать более сложный химико-минералогический состав кометной пыли, включающий в себя льды и силикаты.
\end{abstract}

APPLICATION OF THE SH-MATRICES METHOD TO SIMULATION OF THE SCATTERING PROPERTIES OF ATMOSPHERELESS CELESTIAL BODIES, by D. V. Petrov, N.N. Kiselev, E.A. Zhuzhulina. Modern problems of scattering of electromagnetic waves by particles comparable with the wavelength are often solved using the method of extended boundary conditions. In principle, this method can be used to study scattering by objects of arbitrary shape. However, often the calculations are rather complicated and require a lot of computer time. In this regard, simplification and acceleration of such calculations is very important task. The Sh-matrix method developed by us makes it possible to efficiently calculate the scattering properties of particles of arbitrary shape, both with an arbitrary size parameter and an arbitrary refractive index $m_{0}$. This method is applicable for the study of cometary atmospheres, as well as any regolith-like media. Simulation of the scattering properties of cometary dust was carried out using random Gaussian particles consisting of organic material. Based on calculations of the phase dependence of the degree of linear polarization, the parameters that best reproduce the observational data of Halley's comet are chosen. It was shown that organic particles well describe the value of the inversion 
angle and the polarimetric slope, but such particles cannot reproduce the magnitude and position of the minimum of the negative polarization branch. It was concluded that it is necessary to consider a more complex chemical-mineralogical composition of cometary dust, which includes ice and silicates.

Ключевые слова: поляриметрия, фазовая зависимость поляризации, матрицы формы, компьютерное моделирование

\section{1 Введение}

Для компьютерного моделирования рассеяния электромагнитного излучения несферическими частицами часто используется метод Т-матриц в его различных модификациях (Мищенко и Тревис, 1994; Мищенко и др., 1996). Суть этого метода, разработанного Ватерманом (1973), состоит в разложении рассеянного частицей электромагнитного излучения в ряд по векторным сферическим волновым функциям и дальнейшем вычислении коэффициентов разложения. В данной работе для моделирования рассеивающих свойств использована разработанная автором модификация метода Т-матриц, именуемая методом матриц формы или же Sh-матриц (Петров и др., 2011, 2012). Данный подход дает возможность серьезно упростить вычисления и производить эффективное усреднение рассеивающих свойств несимметричных частиц с произвольным показателем преломления $m_{0}$.

\section{2 Метод матриц формы}

Рассмотрим плоскую электромагнитную волну $\mathbf{E}_{i n c}$, падающую на одиночную однородную частицу неправильной формы, обладающую показателем преломления $m_{0}$. Рассеянное поле обозначим $\mathbf{E}_{s c a}$. Будем описывать нашу волну в сферической системе координат, где любая точка пространства задается радиус-вектором или тремя координатами: $\mathbf{r}=(\rho, \theta, \phi)$, где $\rho$ - расстояние от центра системы координат, $\theta$ и $\phi-$ полярный и азимутальный угол, соответственно, в сферической системе координат с началом в центре частицы (эти координаты характеризуют геометрию светорассеяния). Рассеивающие свойства частицы, зависящие от соотношения ее размера к длине волны, будем характеризовать величиной так называемого размерного параметра, который определяется следующим образом: $\mathrm{X}=2 \pi \mathrm{r} / \lambda$. Здесь $\mathrm{r}$ - характерный размер частицы, а $\lambda$ - длина волны падающего излучения. Выберем начало системы координат внутри частицы. При этом в данной системе координат поверхность неправильной частицы можно описать некоторой функцией $R(\theta, \phi)$. Основная идея метода Т-матриц заключается в разложении падающего и рассеянного полей в ряд по векторным сферическим волновым функциям (Мищенко и др., 2002):

$$
\begin{gathered}
E_{i n c}(\rho, \theta, \phi)=\sum_{n=1}^{\infty} \sum_{m=-n}^{n} a_{m n} R g \mathbf{M}_{m n}+b_{m n} R g \mathbf{N}_{m n} \\
E_{s c a}(\rho, \theta, \phi)=\sum_{n=1}^{\infty} \sum_{m=-n}^{n} p_{m n} \mathbf{M}_{m n}+q_{m n} \mathbf{N}_{m n}
\end{gathered}
$$

где $\mathbf{M}_{m n}, \mathbf{N}_{m n}, R g \mathbf{M}_{m n}, R g \mathbf{N}_{m n}$ - векторные сферические волновые функции, а $a_{m n}, b_{m n}, p_{m n}, q_{m n}-$ соответствующие им коэффициенты разложения. Функции $R g M_{m n}$ и $R g N_{m n}$ конечны в начале координат (Rg означает “regular” (Мищенко и др., 2002)). Явные выражения для векторных сферических волновых функций приведены, например, в работе (Киселев, Чернова, 1976). Коэффициенты разложения рассеянного поля $p_{m n}, q_{m n}$ связаны с коэффициентами разложения падающего поля $a_{m n}, b_{m n}$ соотношениями, следующими из линейности уравнений Максвелла:

$$
p_{m n}=\sum_{n^{\prime}=1}^{\infty} \sum_{m^{\prime}=-n^{\prime}}^{n^{\prime}} \mathbf{T}_{m n m^{\prime} n^{\prime}}^{11} a_{m^{\prime} n^{\prime}}+\mathbf{T}_{m n m^{\prime} n^{\prime}}^{12} b_{m^{\prime} n^{\prime}}
$$




$$
q_{m n}=\sum_{n^{\prime}=1}^{\infty} \sum_{m^{\prime}=-n^{\prime}}^{n^{\prime}} \mathbf{T}_{m n m^{\prime} n^{\prime}}^{21} a_{m^{\prime} n^{\prime}}+\mathbf{T}_{m n m^{\prime} n^{\prime}}^{22} b_{m^{\prime} n^{\prime}}
$$

Матрица $T_{m n m^{\prime} n^{\prime}}=\left(T_{m n m^{\prime} n^{\prime}}^{11} ; T_{m n m^{\prime} n^{\prime}}^{12} ; T_{m n m^{\prime} n^{\prime}}^{21} ; T_{m n m^{\prime} n^{\prime}}^{22}\right)$, связывающая эти два набора коэффициентов, называется Т-матрицей. Матрица $T_{m n m^{\prime} n^{\prime}}$ зависит только от физических и геометрических характеристик рассеивающей частицы, таких как размерный параметр, форма, относительный показатель преломления, и не зависит от геометрии освещения/наблюдения и состояния поляризации падающего света. Не так давно был разработан метод матриц формы (Sh-матриц), которые зависят только от формы частицы и позволяют вычислить Т-матрицу частицы данной формы для любого значения размерного параметра и показателя преломления (Петров и др., 2011, 2012), а следовательно, определить любую характеристику рассеянного излучения в любой точке пространства.

В настоящее время большинство исследований сосредоточено на изучении интенсивности и степени линейной поляризации рассеянного света в дальней зоне как функций угла рассеяния. Для таких астрономических объектов, как планеты, астероиды и кометы, целесообразно рассматривать степень поляризации и позиционный угол плоскости поляризации по отношению к плоскости, ортогональной к плоскости рассеяния. Степень линейной поляризации определяется соотношением:

$$
P(\alpha)=\frac{I_{s}-I_{p}}{I_{s}+I_{p}}
$$

где $I_{s}$ и $I_{p}$ - компоненты интенсивности рассеянного излучения, относящиеся к плоскости, ортогональной к плоскости рассеяния, и к самой плоскости рассеяния соответственно. Плоскость рассеяния определяется двумя векторами: Солнце - небесное тело и небесное тело - Земля. Наблюдаемая интенсивность и степень линейной поляризации рассеянного излучения зависит от фазового угла $\alpha$. Фазовый угол является углом между направлениями Солнце- объект и объект-наблюдатель. Значение степени линейной поляризации формально становится отрицательным, если преимущественное направление колебаний электрического вектора лежит в плоскости рассеяния. Теория (Феофилов, 1959) предсказывает отрицательную поляризацию рассеянного излучения для ряда случаев. Поляризация излучения, рассеянного рэлеевскими частицами, всегда является положительной. Излучение, рассеянное на френелевской поверхности, также всегда поляризовано положительно. Рассеяние на пылевых частицах, чьи размеры порядка длины волны, проявляет отрицательную поляризацию на малых фазовых углах $(\alpha<25)$ и положительную поляризацию на больших фазовых углах. Для удаленных космических объектов, таких как Луна, астероиды и спутники планет, это явление известно с 1929 г. (Лио, 1929), для комет - с 1976 г. (Киселев, Чернова, 1976).

Фазовые зависимости поляризации предоставляют хорошую возможность изучать физические свойства рассеивающих газопылевых сред и поверхностей. Основной метод, используемый для этих целей, состоит в подборе таких параметров модели ансамбля рассеивающих частиц, при которых расчетные фазовые кривые поляризации полностью или частично совпадают с фазовыми зависимостями, полученными из наблюдений. Отсюда видно, насколько важными являются как разработка теоретических моделей, так и полнота и надежность наблюдательных данных.

\section{3 Моделирование рассеивающих свойств кометной пыли}

Моделирование рассеивающих свойств кометной пыли заключается в выборе параметров модельных частиц (форма, размеры, показатель преломления), которые позволят воспроизвести как можно большее количество вышеприведенных параметров, полученных в результате наблюдений. Теория Ми, описывающая рассеяние света сферической частицей, неприменима для интерпретации наблюдательных данных, поскольку из-за “правильной” формы рассеивающего объекта в фазовой картине рассеяния наблюдается множество интерференционных максимумов и минимумов. Наблюдаемые фазовые кривые комет такими особенностями не обладают, и изучения кометной пыли in situ (в ходе миссии “Розетта") показали, что частицы кометной пыли обладают сложной, несферической формой 


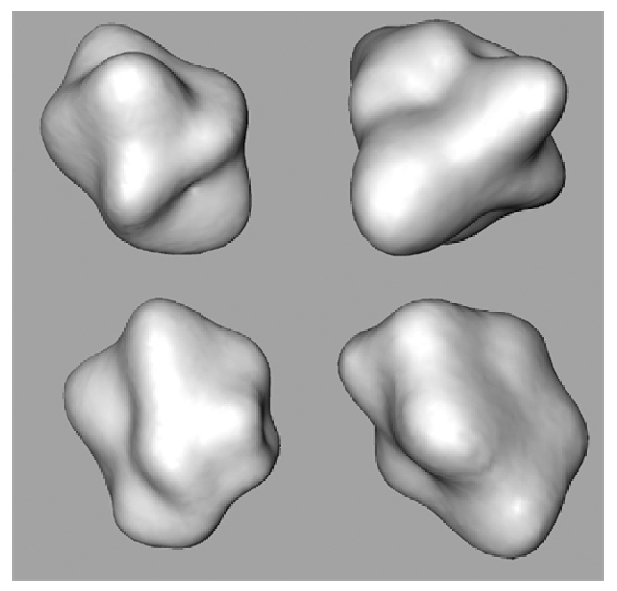

Рис. 1. Примеры случайных гауссовских частиц

(Ланжевин и др., 2016). Один из самых удачных способов математического описания частиц достаточно неправильной формы, воспроизводящих реальные частицы кометной пыли, предложил Муйнонен (1996). Им была создана математическая модель так называемых гауссовских неправильных частиц, которые стали достаточно широко использоваться при моделировании рассеивающих свойств космических объектов (Муйнонен и др., 2007). Примеры случайных гауссовских частиц приведены на рис. 1.

Фазовая зависимость поляризации излучения, рассеянного на пылевых частицах, характеризуется шестью параметрами:

$P_{\text {min }}, \alpha_{\text {min }}-$ параметры, которые задают величину и положение минимума отрицательной ветви фазовой зависимости поляризации;

$\alpha_{0}$ - угол инверсии, при котором поляризация меняет знак, то есть угол, начиная с которого колебания электрического вектора лежат преимущественно в плоскости, перпендикулярной плоскости рассеяния;

$\mathrm{h}$ - поляриметрический наклон, который определяет крутизну восходящей ветви поляризационной зависимости. Согласно определению (Дольфюс и др., 1989), наклон есть производная фазовой функции поляризации в точке инверсии $(\mathrm{h}=\mathrm{dP} / \mathrm{d} \alpha)$;

$P_{\max }, \alpha_{\max }-$ параметры, которые описывают величину и положение максимума положительной ветви фазовой зависимости поляризации.

Кометные пылинки содержат органику. В работе (Валлис и др., 1988) были исследованы параметры таких органических частиц и сделан вывод о том, что их показатель преломления в “красной” области спектра может быть равен $m_{0}=1.4+0.156 i$. Мы провели модельные расчеты степени линейной поляризации для случайных гауссовских частиц с вышеупомянутым показателем преломления для различных размеров и значений фазового угла. Карта степени поляризации приведена на рис. 2 . Здесь по оси абсцисс отложен фазовый угол, а по оси ординат - размерный параметр частицы Х. Значения степени поляризации выделены цветом, причем светлые участки соответствуют высоким значениям степени поляризации, а темные - низким. Из данного рисунка видно, что степень поляризации на больших и малых размерах частиц достаточно похожа и представляет собой пик положительной степени поляризации. На средних же размерах степень поляризации достигает глубоких минимумов.

Нами было проведено моделирование данных наблюдений кометы Галлея в красной области спектра, выполненных в работе (Чернова и др., 1993) (кружочки на рис. 3). Для этого мы использовали усреднение частиц по размерам в диапазоне $X_{\min } \ldots X_{\max }$ по степенному закону $X^{-n}$. Соответственно, моделирование заключалось в подборе таких параметров $X_{\min }, X_{\max }$ и $n$, которые наилучшим образом воспроизведут наблюдательные данные. Сплошная линия на рис. 3 показывает результат 


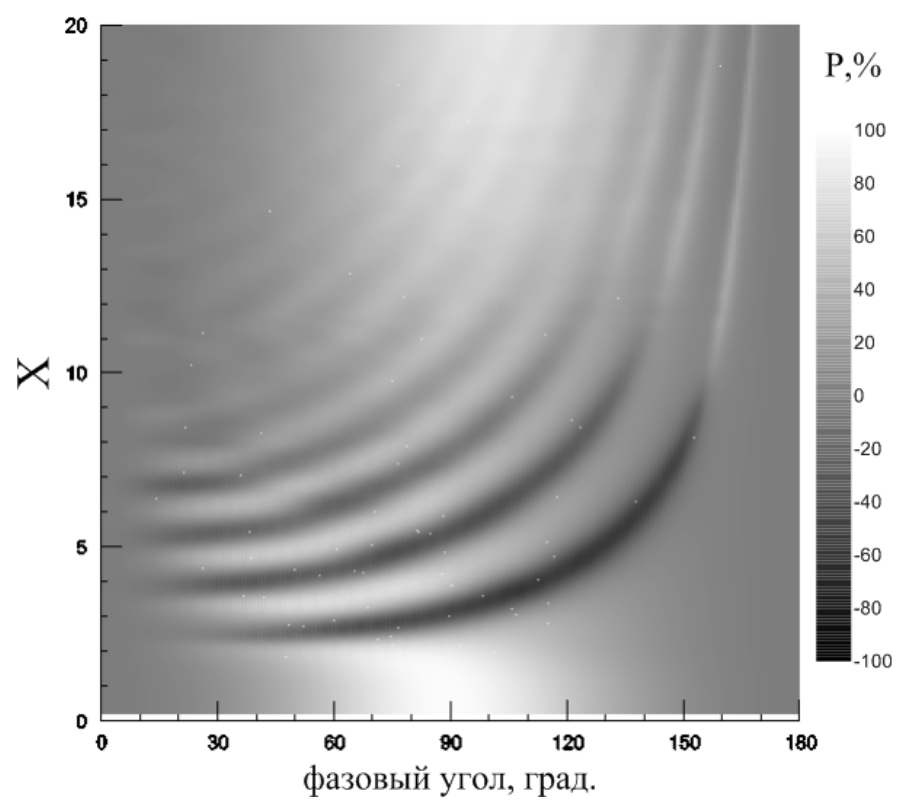

Рис. 2. Карта распределения поляризации света, рассеянного случайными гауссовскими частицами с показателем преломления $m_{0}=1.4+0.156 i$, как функция размерного параметра и фазового угла

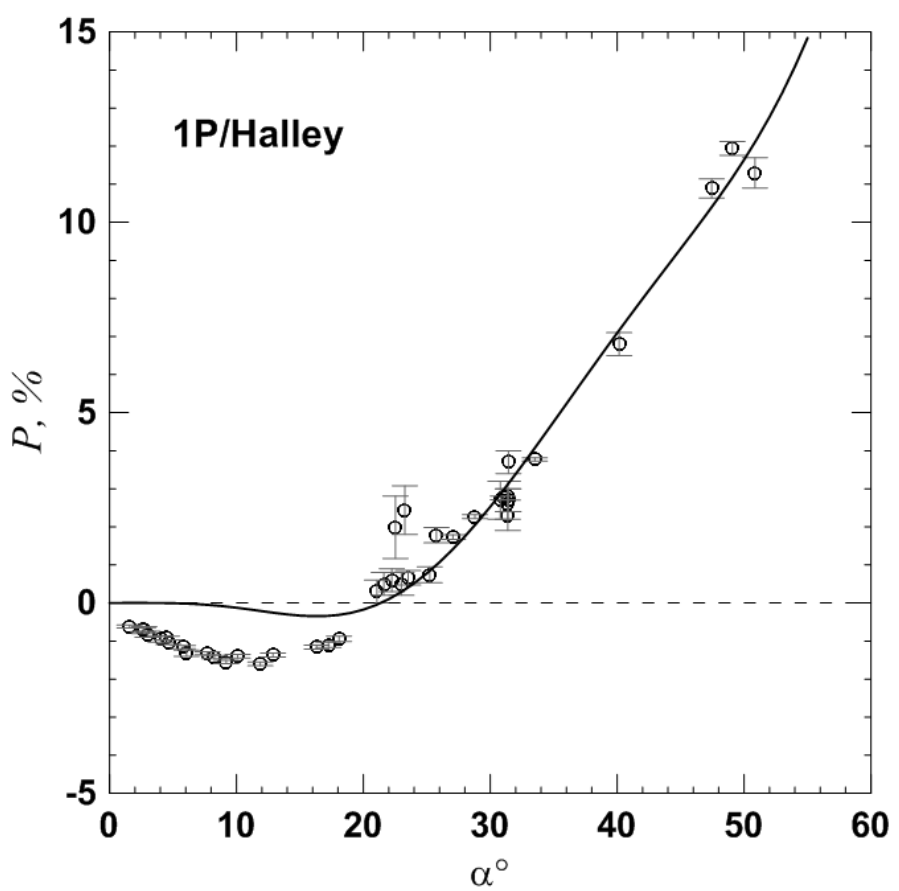

Рис. 3. Наблюдаемая зависимость степени поляризации от фазового угла для кометы Галлея (кружочки), взятая из работы (Чернова и др., 1993). Сплошная линия соответствует наилучшему приближению, достигнутому путем подбора параметров модели органических частиц кометной пыли 
наилучшей подгонки, соответствующей параметрам $X_{\min }=4.1 ; X_{\max }=14.7$ и $n=5.3$. Как видно из рисунка, частицы из органики могут достаточно хорошо воспроизвести значение угла инверсии и поляриметрического наклона, однако величину и положение минимума отрицательной ветви фазовой зависимости степени поляризации частицы из органики не воспроизводят. Следовательно, кометы не могут состоять исключительно из органических частиц, и необходимо учитывать наличие в них частиц иного химического состава, таких как льды и силикаты.

\section{4 Выводы}

В данной работе описан метод расчета рассеивающих свойств удаленных космических объектов. Данный метод применим для изучения кометных атмосфер, а также любых реголитоподобных сред. Проведено моделирование рассеивающих свойств кометной пыли с использованием случайных гауссовских частиц, состоящих из органики. На основании расчетов фазовой зависимости степени линейной поляризации подобраны параметры, наилучшим образом воспроизводящие наблюдательные данные кометы Галлея. Установлено, что органические частицы достаточно хорошо описывают значение угла инверсии и поляриметрического наклона, однако величину и положение минимума отрицательной ветви такие частицы воспроизвести не могут. На основании этого сделан вывод о необходимости рассматривать более сложный химико-минералогический состав кометной пыли, включающий в себя льды и силикаты.

\section{Литература}

Валлис и др. (Wallis M.K., Rabilizirov R., Wickramasinghe N.C.) // Exploration of Halley's Comet / Eds Grewing M., Praderie F., Reinhard R. Springer, Berlin, Heidelberg. 1988. DOI: https://doi.org/10.1007/978-3-642-82971-0_137.

Ватерман (Waterman P.C.) // Computer Techniques for Electromagnetics. Pergamon Press. 1973. P. 97. Дольфюс и др. (Dollfus A., Wolff M., Geake J.E., et al.) // Asteroids II. / Eds Binzel R.P., Gehrels. T. Tucson: Univ. Arizona Press (USA). 1989. P. 596.

Киселев Н.Н., Чернова Г.П. // Астрон. циркуляр. 1976. № 931. С. 5.

Ланжевин и др. (Langevin Y., Hilchenbach M., Ligier N., Merouane S., Hornung K., et al.) // Icarus. 2016. V. 271. P. 76.

Лио (Lyot B.) // Recherches sur la polarisation de la lumière des planètes et de quelques substances terrestres. Annales de l'Observatoire de Paris. Section de Meudon. Orléans: H. Tessier. 1929. V. 8. № 1 .

Мищенко и др. (Mishchenko M.I., Travis L.D., Mackowski D.W.) // J. Quant. Spectr. Radiat. Transfer. 1996. V. 55. Issue 5. P. 535.

Мищенко и др. (Mishchenko M.I., Travis L.D., Lacis A.A.) // Scattering, absorption, and emission of light by small particles. Cambridge: Cambridge University Press. 2002. P. 690.

Мищенко и Тревис (Mishchenko M.I., Travis L.D.) // Applied Optics. 1994. V. 33. Issue 30. P. 7206.

Муйнонен (Muinonen K.) // Earth Moon Planets. 1996. V. 72. P. 339.

Муйнонен и др. (Muinonen K., Zubko E., Tyynela J., Shkuratov Yu., Videen G.) // J. Quant. Spectr. Radiat. Transfer. 2007. V. 106. P. 360.

Петров и др. (Petrov D., Shkuratov Yu., Videen G.) // J. Quant. Spectr. Radiat. Transfer. 2011. V. 112. Issue 11. P. 1636.

Петров и др. (Petrov D., Shkuratov Yu., Videen G.) // J. Quant. Spectr. Radiat. Transfer. 2012. V. 113. P. 2406.

Феофилов П.П. // Поляризованная люминесценция атомов, молекул и кристаллов. М.: ГИФМЛ. 1959.

Чернова и др. (Chernova G.P., Kiselev N.N., Jockers K.) // Icarus. 1993. V. 103. N. 1. P. 144. 